Masero Moreno, I.C., Camacho Peñalosa, M.E. \& Vázquez Cueto, M.J. (2018). Cómo evaluar conocimientos y competencias en la resolución matemática de problemas en el contexto económico a través de rúbricas. Revista Electrónica Interuniversitaria de Formación del Profesorado, 21(1), 51-64.

DOI: http://dx.doi.org/10.6018/reifop.21.1.277981

\title{
Cómo evaluar conocimientos y competencias en la resolución matemática de problemas en el contexto económico a través de rúbricas
}

\author{
Inmaculada C. Masero Moreno, $M^{a}$ Enriqueta Camacho Peñalosa, $M^{a}$ José Vázquez Cueto \\ Universidad de Sevilla
}

\section{Resumen}

El uso de nuevos instrumentos para la evaluación de conocimientos y competencias es un reto en la Educación Matemática a nivel universitario. Dentro del marco teórico de este artículo se abordan las rúbricas como una de las herramientas más adecuada para realizar una evaluación formativa. Esta adquiere sentido si se realizan tareas que faciliten tanto el aprendizaje como la evaluación. En este trabajo, se expone el perfil de las tareas de aprendizaje-evaluación a través de la resolución matemática de problemas en el contexto económico. A partir de las relaciones entre los conocimientos y las competencias a evaluar, se diseña una rúbrica en la que se detallan los criterios de evaluación y sus respectivos indicadores de logro para su uso como herramienta pedagógica. La ventaja de esta propuesta es que puede ser adaptada a cualquier otro contexto.

\section{Palabras clave}

Rúbricas; tareas; competencias; Matemáticas

\section{How to assess knowledge and skills in mathematical problem solving in economic context through rubrics}

\section{Abstract}

The use of new tools for knowledge and skills assessment is currently a real challenge for Mathematical Education. In the theoretical framework of this work, we tackle rubrics as 
one of the most appropriate tools for formative assessment. This makes sense if tasks make students learn and at the same time they assess their performance. In this paper, we expose the characteristics of learning-assessment tasks through mathematical problemsolving in an economic context. We have designed a rubric as a pedagogic tool to assessment skills and knowledge, detailing evaluation criteria and indicators of degrees of labels. These proposals can be adapted to any other contexts.

\section{Key words}

Rubrics; tasks; skills; Mathematics

\section{Introducción}

El concepto de evaluación ha evolucionado desde ser entendida simplemente como "medida" con un enfoque conductista hasta su definición actual como valoración del grado de adquisición de competencias a través de resultados. Como afirman Castillo y Cabrerizo (2009) "medir es condición necesaria para evaluar, pero no suficiente" (p. 24), por lo que evaluar es más complejo ya que implica una interpretación de las medidas referenciadas a una serie de criterios relacionados con los objetivos.

En el ámbito universitario Delgado, Borge, García, Oliver y Salomón (2003) definen la evaluación como "un proceso sistemático de obtener información objetiva y útil en la que apoyar un juicio de valor sobre el diseño, la ejecución y los resultados de la formación con el fin de servir de base para la toma de decisiones" (p.38).

Actualmente, en el contexto del aprendizaje enfocado al desarrollo de competencias, la evaluación hace referencia a los contenidos, las habilidades, las actitudes y las estrategias de aprendizaje.

Evaluar competencias puede identificarse con "reconocer la capacidad de un alumno para dar respuesta a situaciones más o menos reales, problemas o cuestiones que tienen muchas probabilidades de llegar a encontrar, aunque es evidente que nunca del mismo modo en que han sido aprendidos" (Zabala y Arnau, 2007, p. 200).

Para Fernández (2010) "la evaluación en la formación de competencias se escalona en un continuo aprendizaje-evaluación” (p. 17). Esto puede conseguirse a través de la evaluación formativa, que se caracteriza por estar integrada e interrelacionada con el proceso de formación, valorando si el alumno ha alcanzado los objetivos planteados referentes a los conocimientos de una materia y a las competencias asociadas a una asignatura. Sin embargo, hay que tener en cuenta que no se debe evaluar la competencia en sí misma, sino su desempeño, es decir, centrarse en los procesos más que en los productos.

La evaluación debe hacer que los alumnos identifiquen las fortalezas y debilidades de las estrategias cognitivas y metacognitivas de su aprendizaje, evaluando las competencias a partir de sus componentes (conceptual, procedimental y actitudinal). Para ello es necesario establecer indicadores y niveles de desarrollo o logro de los mismos, especificando sus características. Uno de los instrumentos más utilizados para realizar este tipo de evaluación son las rúbricas o matrices de valoración.

\section{Las rúbricas}

La matriz de valoración o rúbrica es una herramienta en la que se expresan las características (criterios relacionados con el aprendizaje de contenidos y desarrollo de competencias) que debe tener el producto final de una tarea propuesta, especificando los niveles de rendimiento que son descritos cualitativamente y/o cuantitativamente. 
Si se realiza la evaluación de una tarea que no tiene asociado una única respuesta o hay varios métodos para resolverla, entonces la evaluación se realiza desde una perspectiva global, y la rúbrica se denomina holística. Si evalúa una tarea con un resultado específico a través de cada una de las componentes de ésta, por ejemplo, la resolución de un problema, entonces la rúbrica es analítica (Mertler, 2001; Torres y Pereda, 2010). Según Alsina (2013) una rúbrica holística se caracteriza por no separar las partes de una tarea y una analítica por evaluar cada parte de una actividad o de un conjunto de actividades. Independientemente del tipo de tarea que se desee valorar y del tema que aborde, las rúbricas deben ajustarse a la evaluación que el docente quiera realizar, fijando los elementos del aprendizaje desarrollado a través de la tarea propuesta y relacionándolos con las competencias que persigue dicho aprendizaje. Partiendo de esos elementos, el docente establece unos criterios de evaluación precisos, claros, consistentes y específicos para cada tarea, que pueden ser organizados en diferentes categorías. A través de estos criterios, el alumno puede verificar en cualquier momento su proceso de aprendizaje.

A continuación, en la matriz de valoración se fijan diferentes niveles de calidad para medir los criterios y valorar el resultado de la tarea. Estos son descritos mediante una escala cualitativa en la que a cada nivel de logro corresponde unas características específicas de rendimiento que constituyen la descripción del dominio asociado.

El número de niveles debe ser adecuado a la complejidad de la tarea, siendo las rúbricas más sencillas aquellas que integran tres o cuatro niveles de logro. Para Espinosa (2013), las más adecuadas son las que admiten valoración central, partiendo de un mínimo de tres niveles y un máximo de cinco con una graduación lógica para que el proceso de evaluación sea eficiente. Esta autora se decanta por distribuir dichos niveles en el intervalo $[0,10]$ según el desempeño de la competencia.

Una opción muy interesante es la que propone García (2014), que aboga por aportar familiaridad al procedimiento estableciendo cuatro niveles basados en las categorías establecidas en las calificaciones universitarias: suspenso (dominio insuficiente de la competencia), aprobado (desempeño aceptable), notable (buen dominio), sobresaliente (nivel de competencia excelente). Esta opción puede facilitar la compresión de la propuesta de evaluación de competencias (criterios e instrumentos) incluida en la guía docente de las asignaturas.

Esta configuración facilita la descomposición de las tareas complejas que integran una competencia en tareas más simples organizadas gradual y operativamente, lo que convierte a las rúbricas en un instrumento idóneo para evaluar competencias (Alsina, 2013).

En resumen, las fases a seguir para elaborar una rúbrica son (McGoldrick y Peterson, 2013):

1.- Identificar las competencias y aquellos elementos de la tarea a los que se asocian.

2.- Fijar los criterios de evaluación y clasificarlos (filas de la matriz de valoración).

3.- Plantear una escala de valoración de los criterios (columnas de la matriz de valoración).

4.- Establecer los descriptores asociados a la escala de valoración en cada uno de los criterios que impliquen el nivel de dominio (celdas de la matriz), cuyo texto no debe ser valorativo sino detallar un comportamiento o una realización.

5.- Validar la rúbrica, comprobando si hay coherencia en los aspectos anteriores en relación con los objetivos de aprendizaje y el contexto (adecuación de la escala, afinidad entre descriptores y los objetivos de evaluación, e identificación de coincidencias entre los criterios y entre los descriptores). 
Una de las ventajas de las rúbricas es su potencial formativo y orientador, pues permite al alumno conocer qué se espera de su trabajo y qué es lo más importante de éste. La evaluación realizada mediante rúbricas es formativa ya que retroalimenta al alumno acerca de sus fortalezas y de los aspectos que debe mejorar, por lo que puede utilizarla como guía y herramienta de autoevaluación. Ello le permite establecer un marco de referencia para conocer el progreso de su trabajo y el desarrollo de su aprendizaje en cualquier momento. Lo anterior implica que el alumno debe disponer de la rúbrica al comienzo de la tarea (Alsina, 2013; Panadero y Jonsson, 2013; Espinosa, 2013; Martínez, Tellado y Raposo, 2013; Gámiz, Torres y Gallego, 2015). Otra ventaja asociada a su uso es que la evaluación es que permite una evaluación imparcial y objetiva (Jonsson, 2014).

Según Cano (2015) uno de los motivos para apostar por las rúbricas es que los alumnos pueden pasar de aplicadores pasivos de los criterios a apropiarse de ellos, si estos y los descriptores son construidos de forma colaborativa con el docente, fomentando así su participación en el proceso de evaluación. La evaluación orientada al aprendizaje puede realizarse a través de una rúbrica, siendo necesario partir de tareas de evaluación que constituyan auténticas tareas de aprendizaje (Ibarra, Rodríguez, y Gómez, 2012).

\section{Tareas de aprendizaje y tareas de aprendizaje-evaluación}

Las tareas son las propuestas de trabajo que hace el docente y las actividades son las acciones que realiza el alumno para poder elaborar lo que cree que se le pide, no existiendo tareas universales que activen todos los tipos de aprendizaje (Goñi, 2008). A la hora de plantear las tareas de aprendizaje no se deben confundir ambos conceptos.

Para Aráez, Bouza, Mateo y Moreno (2011) el concepto de tarea en el contexto educativo es la forma en la que se ordenan las actividades de aprendizaje con el fin de que los alumnos tengan experiencias útiles. Estos autores recogen las cuatro componentes que deben existir en toda tarea de aprendizaje (en Gimeno, 1988):
1.- Finalidad (objetivos del aprendizaje).
2.- Actividades (acciones para desarrollar la tarea).
3.- Recursos (materiales y fuentes de información).
4.- Producto (resultado de las actividades integradas en la tarea).

Además, se pueden incluir como elementos de la tarea: los roles (relaciones entre los alumnos), el escenario donde se desarrolla, el seguimiento y el feedback (Roca, Valcárcel y Verdú, 1990).

En el contexto de la enseñanza de las Matemáticas, Herbst (2012) define la tarea como unidades de significado determinadas en la observación del trabajo matemático que se realiza en el aula, constituyendo un contexto práctico en el que los alumnos razonan sobre las herramientas matemáticas asociadas a un problema. Para este autor, la tarea planteada debe encaminarse a que los alumnos trabajen generando conocimiento.

La clasificación de tareas que propone el proyecto PISA (Ministerio de Educación, Cultura y Deporte, 2013), atendiendo a los tipos de aprendizajes de las Matemáticas de menor a mayor dificultad, es la siguiente:

Tareas asociadas al aprendizaje relacionado con la reproducción denominados ejercicios, en cuya resolución se emplean expresiones y fórmulas. Son necesarias para desarrollar habilidades asociadas a las competencias de bajo nivel e imprescindibles para poder desarrollar otras de nivel superior.

Tareas asociadas al aprendizaje relacionado con la conexión (relación) para cuya resolución se interpreta la información, se identifican elementos y se utilizan relaciones entre conceptos. 
Tareas asociadas al aprendizaje con la reflexión (producción) implican la utilización del pensamiento creativo y de la capacidad de justificar y argumentar la estrategia elegida. Se aplica en la resolución de problemas.

Evidentemente, cada tipo de tarea está relacionado con un tipo de conocimiento y proceso cognitivo. Así, las cuestiones pueden ser utilizadas para valorar el grado de conocimiento sobre los contenidos y la comprensión de los conceptos impartidos, mientras que los ejercicios permiten comprobar la habilidad del alumno en el manejo de las herramientas matemáticas. Por otro lado, los problemas se emplean para saber si el alumno es capaz de relacionar y aplicar determinados conceptos matemáticos en diferentes contextos.

Fernández (2010) afirma que, en la evaluación en formación de competencias, las tareas para el aprendizaje y la evaluación han de ser similares. Bajo esta perspectiva, la evidencia del logro del aprendizaje se debe realizar a través del producto final de las tareas de aprendizaje-evaluación que permite valorar la adquisición y transformación del conocimiento (evidencias del producto).

En el caso de las Matemáticas, las tareas de aprendizaje-evaluación más adecuadas son las que aplican la resolución de problemas. Estas han de partir de situaciones, más o menos reales, en las que el alumno actúa para resolver un problema o una cuestión. Zabala y Arnau (2007) denominan situación-problema a este tipo de tarea.

Por ejemplo, su planteamiento en el contexto económico debe estar orientado a que el alumno transforme un enunciado económico en un problema matemático, identifique la herramienta matemática que debe usar, la aplique, interprete los resultados, y a partir de estos, sea capaz de extraer conclusiones.

Para este tipo de tareas se propone en este trabajo una rúbrica para realizar una evaluación formativa y orientada al aprendizaje en las asignaturas de Matemáticas, en particular para los estudios de tipo económico.

\section{Rúbrica para la evaluación de una situación-problema}

La evaluación en las asignaturas de Matemáticas en el contexto económico suele estar centrada en la comprensión y aplicación de conocimientos y procedimientos matemáticos en la resolución de problemas. Sin embargo, lo adecuado es evaluar si el alumno es competente, es decir, si es capaz de responder de forma eficiente ante una situación concreta planteada en un determinado contexto económico mediante el uso de técnicas matemáticas.

Partiendo de esta premisa, lo ideal es proponer una tarea de aprendizaje-evaluación que implique la resolución de un problema matemático a partir de un enunciado contextualizado en la Economía y la Empresa (situación-problema). Su desarrollo debe proporcionar información sobre el conocimiento o dominio asociado a las distintas componentes de determinadas competencias. Estas componentes han de estar relacionadas con las estrategias que el alumno utiliza para resolver la tarea.

Para evaluar una situación-problema mediante una rúbrica hay que identificar aquellos aspectos, detalles y elementos que han de estar presentes en su resolución. En nuestro caso, deben estar relacionados con los contenidos económicos y los procedimientos matemáticos que utiliza el alumno en la resolución y que deben aparecer reflejados en el producto final de la tarea.

Así, los criterios básicos que consideramos más adecuados para poder valorar el logro de objetivos (conceptos y procedimientos) son los siguientes:

1.- Identificar el concepto y/o fenómeno económico en términos matemáticos. 
2.- Analizar los conceptos y fenómenos económicos en términos matemáticos.

3.- Plantear el problema en términos matemáticos.

4.- Elegir la herramienta matemática.

5.- Aplicar una estrategia adecuada para resolver problemas económicos en términos matemáticos de forma eficiente.

6.- Delimitar los pasos del proceso (resolución).

7.- Obtener la solución.

8.- Establecer relaciones entre los términos matemáticos y económicos.

9.- Interpretar los resultados en términos económicos.

10.- Enunciar conclusiones.

Estos pueden desglosarse para afinar la evaluación hasta el nivel que el docente desee, asignándole la escala de valoración que considere conveniente y un descriptor a cada nivel de logro. Nuestra recomendación es el uso simultáneo de una escala cualitativa y cuantitativa, con un máximo de cinco niveles dependiendo de la dificultad de la situaciónproblema. Esta valoración permite al alumno detectar los aciertos y los errores, facilitando la autoevaluación y detección de los logros, las dificultades y el nivel de rendimiento.

En el ámbito económico, las rúbricas utilizadas en la mayoría de los casos evalúan solo el conocimiento teórico y práctico (McGoldrick y Peterson, 2013; Buttet y Dólar, 2014), siendo menos frecuente compaginar esta rúbrica con otra para evaluar el desarrollo de determinadas competencias (Domínguez, Martín, Paralera, Romero y Tenorio, 2015).

En este trabajo, planteamos una rúbrica para evaluar tanto el dominio de los contenidos teóricos y prácticos como el desarrollo de competencias estableciendo relaciones entre estos aspectos. Para ello, nos basamos en la identificación que realizan Zabala y Arnau (2007, p. 202) sobre aquellas acciones desarrolladas en el proceso de resolución de una situación-problema que permiten evaluar la actuación competente del alumno (Figura 1).

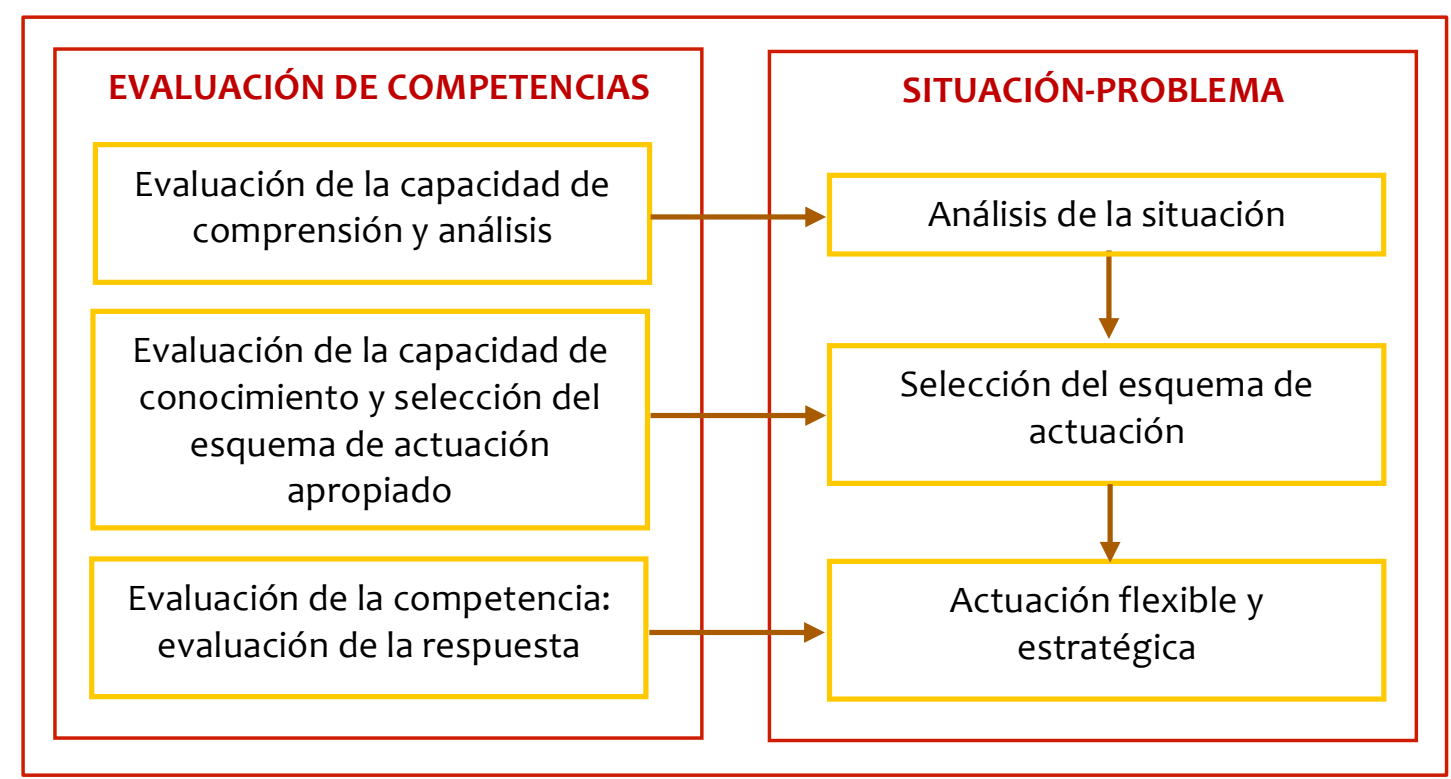

Figura 1: Evaluación en el proceso de actuación competente 

rúbricas

A partir de este esquema, proponemos los criterios asociados a cada competencia para evaluar una situación-problema en el contexto económico.

Tabla 1.

Criterios de evaluación de la actuación competente en la resolución de problemas matemáticos en el contexto económico

\begin{tabular}{|c|c|c|}
\hline COMPETENCIAS & CRITERIOS & $\begin{array}{l}\text { UNIDAD EN LA QUE SE } \\
\text { VALORA: } \\
\text { SITUACIÓN-PROBLEMA }\end{array}$ \\
\hline \multirow{3}{*}{$\begin{array}{l}\text { Capacidad de comprensión y } \\
\text { análisis }\end{array}$} & $\begin{array}{l}\text { Identificación de los conceptos } \\
\text { y/o fenómenos económicos en } \\
\text { términos matemáticos }\end{array}$ & \multirow{3}{*}{ Análisis de la situación } \\
\hline & $\begin{array}{l}\text { Análisis de los conceptos y } \\
\text { fenómenos económicos en } \\
\text { términos matemáticos }\end{array}$ & \\
\hline & $\begin{array}{l}\text { Planteamiento del problema en } \\
\text { términos matemáticos }\end{array}$ & \\
\hline \multirow{4}{*}{$\begin{array}{l}\text { Capacidad de conocimiento } \\
\text { y selección del esquema de } \\
\text { actuación apropiado }\end{array}$} & $\begin{array}{l}\text { Elección de la herramienta } \\
\text { matemática }\end{array}$ & \multirow{4}{*}{$\begin{array}{l}\text { Selección esquema de } \\
\text { actuación }\end{array}$} \\
\hline & $\begin{array}{l}\text { Aplicación de la herramienta } \\
\text { matemática }\end{array}$ & \\
\hline & Etapas de la resolución & \\
\hline & Obtención de la solución & \\
\hline \multirow{3}{*}{ Evaluación de la respuesta } & $\begin{array}{l}\text { Interpretar los resultados en } \\
\text { términos económicos }\end{array}$ & \multirow{3}{*}{$\begin{array}{l}\text { Actuación flexible y } \\
\text { estratégica }\end{array}$} \\
\hline & $\begin{array}{l}\text { Establecer relaciones entre los } \\
\text { términos matemáticos y } \\
\text { económicos }\end{array}$ & \\
\hline & Enunciar conclusiones & \\
\hline
\end{tabular}

Una vez fijados los criterios, establecemos los indicadores que aparecen recogidos en la rúbrica siguiente.

Tabla 2.

Rúbrica de evaluación de la actuación competente en la resolución de problemas matemáticos en el contexto económico

\begin{tabular}{|c|c|c|c|c|c|}
\hline \multirow{4}{*}{ 足 } & & CRITERIOS & Mal & Aceptable & Bien \\
\hline & \multirow{3}{*}{ 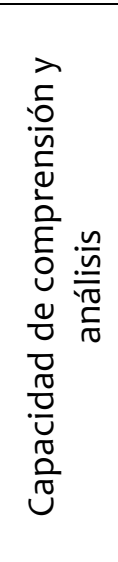 } & $\begin{array}{l}\text { Identificar conceptos } \\
\text { y fenómenos } \\
\text { económicos en } \\
\text { términos } \\
\text { matemáticos }\end{array}$ & $\begin{array}{l}\text { No identifica } \\
\text { correctamente } \\
\text { los conceptos } \\
\text { y/o fenómenos }\end{array}$ & $\begin{array}{l}\text { Identifica } \\
\text { algunos } \\
\text { conceptos y/o } \\
\text { fenómenos }\end{array}$ & $\begin{array}{l}\text { Identifica } \\
\text { todos los } \\
\text { conceptos y } \\
\text { fenómenos }\end{array}$ \\
\hline & & $\begin{array}{l}\text { Analizar los } \\
\text { conceptos y } \\
\text { fenómenos } \\
\text { económicos en } \\
\text { términos } \\
\text { matemáticos } \\
\end{array}$ & $\begin{array}{l}\text { No establece } \\
\text { las relaciones } \\
\text { necesarias para } \\
\text { plantear el } \\
\text { problema }\end{array}$ & $\begin{array}{l}\text { Establece } \\
\text { algunas de las } \\
\text { relaciones } \\
\text { necesarias } \\
\text { para plantear } \\
\text { el problema }\end{array}$ & $\begin{array}{l}\text { Establece } \\
\text { todas las } \\
\text { relaciones } \\
\text { necesarias } \\
\text { para plantear } \\
\text { el problema }\end{array}$ \\
\hline & & Plantear el problema & No plantea el & Plantea parte & Plantea el \\
\hline
\end{tabular}




\begin{tabular}{|c|c|c|c|c|}
\hline & $\begin{array}{l}\text { en términos } \\
\text { matemáticos }\end{array}$ & $\begin{array}{l}\text { problema } \\
\text { matemático } \\
\text { adecuado }\end{array}$ & $\begin{array}{l}\text { del problema } \\
\text { matemático }\end{array}$ & $\begin{array}{l}\text { problema } \\
\text { matemático }\end{array}$ \\
\hline \multirow{3}{*}{ 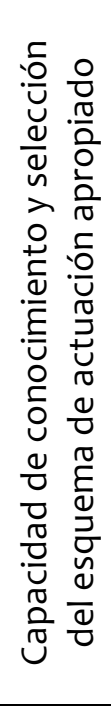 } & $\begin{array}{l}\text { Elegir la herramienta } \\
\text { matemática }\end{array}$ & $\begin{array}{l}\text { No identifica el } \\
\text { tipo de } \\
\text { herramienta }\end{array}$ & $\begin{array}{l}\text { Identifica la } \\
\text { herramienta } \\
\text { pero no el } \\
\text { tipo } \\
\text { adecuado al } \\
\text { problema }\end{array}$ & $\begin{array}{l}\text { Identifica el } \\
\text { tipo de } \\
\text { herramienta }\end{array}$ \\
\hline & $\begin{array}{l}\text { Etapas de la } \\
\text { resolución }\end{array}$ & $\begin{array}{l}\text { Resolución sin } \\
\text { objetivo y no } \\
\text { organizada }\end{array}$ & $\begin{array}{l}\text { Esquema con } \\
\text { desarrollo } \\
\text { básico de las } \\
\text { etapas }\end{array}$ & $\begin{array}{l}\text { Esquemas de } \\
\text { etapas con } \\
\text { exposición } \\
\text { lógica de } \\
\text { contenido }\end{array}$ \\
\hline & Obtener la solución & $\begin{array}{l}\text { No obtiene la } \\
\text { solución o es } \\
\text { errónea }\end{array}$ & $\begin{array}{l}\text { Obtiene la } \\
\text { solución con } \\
\text { algún error no } \\
\text { significativo }\end{array}$ & $\begin{array}{l}\text { Obtiene la } \\
\text { solución }\end{array}$ \\
\hline \multirow{3}{*}{ 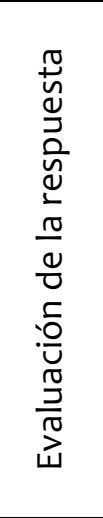 } & $\begin{array}{l}\text { Establecer relaciones } \\
\text { entre los términos } \\
\text { matemáticos y } \\
\text { económicos } \\
\end{array}$ & $\begin{array}{l}\text { Relaciones } \\
\text { erróneas }\end{array}$ & $\begin{array}{l}\text { Establece las } \\
\text { relaciones de } \\
\text { forma } \\
\text { esquemática }\end{array}$ & $\begin{array}{l}\text { Establece y } \\
\text { explica todas } \\
\text { las relaciones }\end{array}$ \\
\hline & $\begin{array}{l}\text { Interpretar los } \\
\text { resultados en } \\
\text { términos económicos }\end{array}$ & $\begin{array}{l}\text { No interpreta o } \\
\text { asigna } \\
\text { significado } \\
\text { erróneo }\end{array}$ & $\begin{array}{l}\text { Interpreta } \\
\text { con algún } \\
\text { error }\end{array}$ & $\begin{array}{l}\text { Interpreta de } \\
\text { forma } \\
\text { coherente }\end{array}$ \\
\hline & Enunciar conclusiones & $\begin{array}{l}\text { No establece } \\
\text { conclusiones o } \\
\text { son erróneas }\end{array}$ & $\begin{array}{l}\text { Establece las } \\
\text { principales } \\
\text { conclusiones }\end{array}$ & $\begin{array}{l}\text { Expone todas } \\
\text { las } \\
\text { conclusiones }\end{array}$ \\
\hline
\end{tabular}

Esta rúbrica permite evaluar tanto el razonamiento matemático conceptual como el formal mediante la resolución de problemas (Hull, Kuo, Gupta, y Elby, 2013).

Puede ser usada como una plantilla para evaluar la tarea de cualquier situación-problema sobre la resolución de un problema matemático en el contexto económico. Solo es necesario adaptarla a cada caso, especificando en cada criterio y nivel cuáles son los contenidos económicos, matemáticos y los procedimientos.

Para evaluar con la rúbrica anterior el nivel de competencia adquirido por el alumno, la escala más adecuada es: aún no es competente, competente y experto. Sin embargo, el docente puede fijar un nivel mínimo en cada criterio o bien priorizar unos sobre otros para calificar en la escala anterior.

Si se alcanza el nivel mínimo, previamente establecido en cada una de las competencias, se puede concluir que también se ha logrado el desarrollo de la capacidad de aplicar el conocimiento a la práctica, competencia no incluida en la tabla anterior.

Para realizar una completa evaluación de la tarea, es importante valorar el informe y/o exposición sobre el trabajo desarrollado. Los aspectos que deben ser evaluados son los relacionados con el formato, la organización, la redacción y los recursos gráficos, que además, pueden ser considerados como criterios asociados a la comunicación escrita. Esto permite valorar la capacidad de realizar presentaciones verbales o escritas en lengua nativa, 
competencia clave genérica del Área de Administración de Empresas (González y Wagenaar, 2006).

En el caso de una presentación oral, se deben valorar aspectos de la exposición como el lenguaje verbal y no verbal o el interés por captar el interés de la audiencia. La tabla siguiente recoge los criterios y la unidad en la que se valoran.

Tabla 3.

Criterios de evaluación de la presentación y la comunicación oral

\begin{tabular}{|l|l|l|}
\hline \multicolumn{1}{|c|}{ COMPETENCIAS } & \multicolumn{1}{|c|}{ CRITERIOS } & UNIDAD EN LA QUE SE VALORA \\
\hline $\begin{array}{l}\text { Capacidad de realizar } \\
\text { presentaciones } \\
\text { verbales y/o escritas }\end{array}$ & $\begin{array}{l}\text { Formato/Elementos } \\
\text { Organización } \\
\text { Redacción } \\
\text { Recursos gráficos } \\
\text { Creatividad }\end{array}$ & Producto final de la tarea \\
\hline Comunicación oral & $\begin{array}{l}\text { Lenguaje verbal y no } \\
\text { verbal } \\
\text { Captación del interés del } \\
\text { público }\end{array}$ & Exposición de la tarea \\
\hline
\end{tabular}

Si la exposición se realiza en grupo, se debe valorar la actuación su actuación incluyendo criterios como la capacidad de los miembros para interactuar entre ellos y con el auditorio. Además, es conveniente realizar la evaluación individual de cada miembro del grupo en la exposición.

A continuación, se expone la rúbrica que proponemos para evaluar las competencias anteriores a partir de una presentación oral o escrita que recoja la resolución de cualquier situación-problema, independientemente del contexto y de la materia, tan solo es necesario adaptar el criterio "organización" al contenido de la tarea.

Tabla 4.

Rúbrica de evaluación de la realización de presentaciones y la comunicación oral

\begin{tabular}{|c|c|c|c|c|c|}
\hline \multirow{3}{*}{ 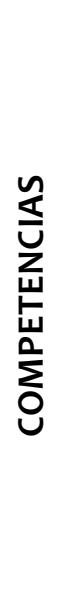 } & & $\begin{array}{c}\text { CRITERIO } \\
\mathrm{S}\end{array}$ & Mal & Aceptable & Bien \\
\hline & \multirow{2}{*}{ 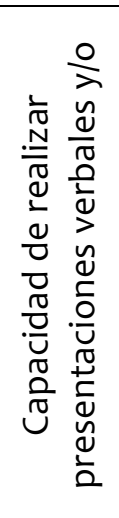 } & Formato & $\begin{array}{l}\text { Ausencia de } \\
\text { portada, índice, } \\
\text { conclusiones y } \\
\text { bibliografía }\end{array}$ & $\begin{array}{l}\text { Ausencia de } \\
\text { índice, } \\
\text { conclusiones o } \\
\text { bibliografía o } \\
\text { bien inadecuados }\end{array}$ & $\begin{array}{l}\text { Portada, índice, } \\
\text { conclusiones y } \\
\text { bibliografía } \\
\text { adecuados }\end{array}$ \\
\hline & & $\begin{array}{l}\text { Organizac } \\
\text { ión }\end{array}$ & $\begin{array}{l}\text { Ausencia de } \\
\text { orden lógico en } \\
\text { algunas etapas de } \\
\text { la resolución y/o } \\
\text { no están } \\
\text { completas }\end{array}$ & $\begin{array}{l}\text { Orden coherente } \\
\text { de las etapas } \\
\text { principales pero } \\
\text { faltan algunos } \\
\text { elementos como } \\
\text { las conclusiones }\end{array}$ & $\begin{array}{l}\text { Secuencia lógica y } \\
\text { ordenada de todas las } \\
\text { etapas de la } \\
\text { resolución están } \\
\text { completas }\end{array}$ \\
\hline
\end{tabular}




\begin{tabular}{|c|c|c|c|c|}
\hline & $\begin{array}{l}\text { Recursos } \\
\text { gráficos }\end{array}$ & $\begin{array}{l}\text { Ausencia de } \\
\text { unidad de estilo } \\
\text { (tipo de letra, } \\
\text { tamaño, estilo y } \\
\text { fondo). Imágenes, } \\
\text { gráficos o tablas } \\
\text { no identificables } \\
\end{array}$ & $\begin{array}{l}\text { Pequeños errores } \\
\text { en la } \\
\text { homogeneidad } \\
\text { de estilo y/o en } \\
\text { las imágenes, } \\
\text { gráficos o tablas }\end{array}$ & $\begin{array}{l}\text { Unidad de estilo que } \\
\text { facilita la lectura y } \\
\text { diferencia los } \\
\text { apartados. Imágenes, } \\
\text { gráficos o tablas } \\
\text { nítidos y de tamaño } \\
\text { adecuado }\end{array}$ \\
\hline & $\begin{array}{l}\text { Ortografía } \\
\text { y } \\
\text { gramática }\end{array}$ & $\begin{array}{l}\text { Errores } \\
\text { ortográficos y } \\
\text { gramaticales que } \\
\text { dificultan la } \\
\text { comprensión } \\
\end{array}$ & $\begin{array}{l}\text { Algún error } \\
\text { ortográfico y/o } \\
\text { gramatical }\end{array}$ & $\begin{array}{l}\text { Ortografía y } \\
\text { gramática correcta }\end{array}$ \\
\hline & $\begin{array}{l}\text { Creativida } \\
\text { d }\end{array}$ & $\begin{array}{l}\text { Presentación } \\
\text { prototipo de } \\
\text { diseño poco } \\
\text { atractivo }\end{array}$ & $\begin{array}{l}\text { Presentación } \\
\text { prototipo con } \\
\text { colores y } \\
\text { formatos } \\
\text { atractivos }\end{array}$ & $\begin{array}{l}\text { Diseño original y } \\
\text { creativo. Incluye } \\
\text { formatos y diferentes } \\
\text { elementos } \\
\text { (imágenes, tablas, } \\
\text { gráficas...) que hacen } \\
\text { más atractiva la } \\
\text { presentación }\end{array}$ \\
\hline 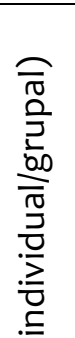 & $\begin{array}{l}\text { Lenguaje } \\
\text { verbal }\end{array}$ & $\begin{array}{l}\text { Ritmo monótono, } \\
\text { oraciones } \\
\text { incompletas y } \\
\text { vocalización } \\
\text { inadecuada }\end{array}$ & $\begin{array}{l}\text { Tono adecuado, } \\
\text { pronunciación } \\
\text { correcta }\end{array}$ & $\begin{array}{l}\text { Pronunciación y ritmo } \\
\text { que refuerzan el } \\
\text { mensaje. Expresión } \\
\text { gramatical clara y } \\
\text { sencilla para facilitar } \\
\text { la comprensión del } \\
\text { oyente }\end{array}$ \\
\hline $\begin{array}{l}\frac{7}{0} \\
\frac{0}{0} \\
: 0 \\
: 0\end{array}$ & $\begin{array}{l}\text { Lenguaje } \\
\text { no verbal }\end{array}$ & $\begin{array}{l}\text { Expresión } \\
\text { corporal y actitud } \\
\text { inadecuados }\end{array}$ & $\begin{array}{l}\text { Expresión } \\
\text { corporal o actitud } \\
\text { adecuados }\end{array}$ & $\begin{array}{l}\text { Expresión corporal y } \\
\text { actitud adecuados }\end{array}$ \\
\hline 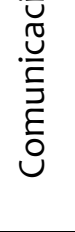 & $\begin{array}{l}\text { Captación } \\
\text { del } \\
\text { interés } \\
\text { del } \\
\text { público }\end{array}$ & $\begin{array}{l}\text { No se dirigen al } \\
\text { público o se } \\
\text { centra en una } \\
\text { parte del mismo o } \\
\text { en el profesor }\end{array}$ & $\begin{array}{l}\text { Se dirigen al } \\
\text { profesor y al } \\
\text { público con } \\
\text { asiduidad }\end{array}$ & $\begin{array}{l}\text { Se dirigen a toda la } \\
\text { audiencia (público y } \\
\text { profesor). Preguntan } \\
\text { y realizan } \\
\text { aclaraciones }\end{array}$ \\
\hline
\end{tabular}

\section{Discusión}

Para diseñar la rúbrica hemos seguido los cinco pasos propuestos por McGoldrick y Peterson (2013), aunque nuestra propuesta está alejada del perfil planteado por estos y por Buttet y Dólar (2014) que evalúan el conocimiento teórico y práctico y no el desarrollo de las competencias asociadas al aprendizaje de este. Sin embargo, coincidimos en el uso de una escala central de tres niveles, como también proponen Domínguez et al. (2015), aunque su escala tiene una calificación numérica en ambas rúbricas.

En este trabajo hemos recogido toda la información necesaria para la creación de la rúbrica que proponemos, hecho que no se incluye en los artículos de la revisión analizada por Marín y Santandreu (2015) sobre la producción científica acerca de las rúbricas.

Además, hemos incluido suficiente información para facilitar la comprobación de su validez (en cada criterio se mide lo que se quiere medir). De acuerdo con Reddy y Andrade (2010) hemos utilizado un lenguaje claro para evitar interpretaciones ambiguas. También hemos 
expuesto la importancia y justificado la necesidad de todos los criterios de la rúbrica (Jonsson y Svingby, 2007), que en este caso representan los aspectos evaluables dentro de la resolución matemática de problemas contextualizados y del trabajo oral y/o escrito.

Dos de los inconvenientes más comunes del uso de las rúbricas, identificados por Reddy y Andrade (2010), son la reticencia que muestran los docentes hacia estas y el tiempo y esfuerzo que conlleva para estos su creación. Nuestra propuesta puede evitarlos, ya que si un docente adapta nuestra rúbrica a la resolución de una situación problema su trabajo se limita a la identificación de los conceptos matemáticos y económicos que se abordan y a la herramienta a utilizar en la resolución. No necesitaría tener ningún tipo de conocimiento especial sobre las tendencias actuales en evaluación, ni dedicarle mucho esfuerzo en tiempo/recursos. Esto haría que la utilización de nuestra rúbrica tuviera un mínimo coste frente a las ventajas que proporciona para evaluar competencias, lo que justificaría su uso según Marín y Santandreu (2015). Incluso la parte dedicada a la evaluación de la presentación oral y/o escrita no necesita adaptación.

La rúbrica propuesta no incluye ejemplos en los niveles de desempeño de la resolución del problema, lo que puede suponer un inconveniente (Jonsson y Svingby, 2007). Con ello perseguimos la amplitud y generalidad que queremos otorgarle como ventaja. Sin embargo, una vez adaptada a un problema particular habría que especificar en cada criterio los aspectos matemáticos y económicos que caracterizan el tipo de problema, con lo que se evitaría este inconveniente. En el caso de la rúbrica para la presentación oral y/o escrita sí se han introducido ejemplos que sirvan de guía tanto al docente como a los estudiantes.

En el diseño y creación de la rúbrica se ha tenido en cuenta el contexto y los objetivos de aprendizaje para asegurar su validez (recomendación recogida por Jonsson y Svingby, 2007; Panadero y Jonsson, 2013; Reddy y Andrade, 2010). Dichos objetivos de aprendizaje están reflejados e introducidos a nivel teórico y práctico en los criterios, que han sido completamente adaptados a la tarea (recomendación de Reddy y Andrade, 2010). La escala de tres niveles también contribuye a mejorar la consistencia de las puntuaciones (Jonsson y Svingby, 2007; Reddy y Andrade, 2010).

Sin embargo, estamos en desacuerdo en la recomendación recogida por las revisiones realizadas por los últimos autores con respecto al uso de rúbricas holísticas para evaluar competencias genéricas. En nuestra rúbrica analítica, creemos que el hecho de reflejar en los criterios todas las dimensiones implícitas de la competencia de resolución de problemas facilita la valoración de su nivel de adquisición.

Marin y Santandreu (2015) corroboran la utilización de las rúbricas para evaluar en diferentes campos, sin embargo, hemos de señalar que en asignaturas de Matemáticas en estudios universitarios son muy escasas. Por lo tanto, creemos que esta propuesta sirve para ampliar su uso al evaluar la adquisición de conocimientos teóricos y prácticos y las competencias adquiridas en la resolución matemática de un problema contextualizado.

\section{Conclusiones}

Este trabajo se ha desarrollado en torno a las tareas y a la evaluación. En él hemos establecido el perfil que deben tener las tareas de aprendizaje-evaluación en las Matemáticas particularizadas para la Economía a través de las denominadas situaciónproblema.

El esquema de evaluación del proceso de actuación competente en la resolución matemática de una situación-problema, particularizada en un contexto económico, nos ha servido de base para establecer una serie de criterios a los que asociar cada competencia. 
Así, hemos recogido las dimensiones básicas de una competencia relacionándolas con los aspectos fundamentales de la resolución de problemas. A partir de ellos construimos una rúbrica con una escala de valoración central y tres niveles. Como se observa, la evaluación ha partido de una reflexión sobre qué se evalúa, extendiéndose en torno a cómo se enseña, ya que el sistema de evaluación debe estar directamente en concordancia con la metodología de aprendizaje.

La rúbrica propuesta presenta ventajas importantes. La primera es que permite la evaluación del conocimiento teórico y práctico junto a la adquisición de competencias. Esto evita tener que elaborar y emplear dos rúbricas diferentes.

Su fin es servir como plantilla para realizar la evaluación de cualquier problema matemático contextualizado, particularizando en los criterios aquellos conceptos y herramientas matemáticas propias de cada situación-problema. Cualquier docente que quiera utilizarla solo tiene que particularizarla para cada tarea, suponiendo una ventaja a la hora de plantear su uso como herramienta para el desarrollo de la evaluación formativa objetiva. De esta forma estamos evitando uno de los puntos con mayor dificultad a hora de su creación como es la identificación de indicadores y la descripción cualitativa de los niveles de valoración, que además han de claros y explícitos.

Esta propuesta respeta la libertad de cada docente a la hora de evaluar, ya que no se han establecido niveles mínimos ni se ha dado preferencia a los criterios para que cada docente imponga sus prioridades en su propia rúbrica.

Si la evaluación de la misma tarea es desarrollada por diferentes docentes, esta opción logra eliminar la subjetividad ya que los criterios empleados son compartidos por todos, facilitando la coordinación.

Por último, en el caso de la evaluación de trabajos, tanto informes como presentaciones, solo es necesario adaptar el criterio que recoge la organización. Y, por lo tanto, puede ser usada para evaluar en cualquier contexto.

Creemos que esta rúbrica puede constituir una herramienta que permita a muchos docentes introducir la evaluación de competencias en las asignaturas de Matemáticas para la Economía y la Empresa.

En resumen, con este trabajo ponemos a disposición de los docentes una rúbrica que permite la evaluación conjunta de conocimientos y competencias en las tareas Matemáticas aplicadas al contexto económico. No obstante, puede ser fácilmente adaptada a cualquier disciplina que utilice las Matemáticas para resolver problemas de otras áreas de conocimiento científico.

\section{Referencias}

Alsina, J. (Coord) (2013). Rúbricas para la evaluación de competencias. Colección Cuadernos de Docencia. Barcelona: Ediciones Octaedro.

Aráez, I., Bouza, M., Mateo, J.M. y Moreno, M.D. (2011). Programación Didáctica de ESPA. Ministerio de Educación. Secretaría General Técnica. Subdirección General de Documentación y Publicaciones.

Buttet, S. \& Dolar, V. (2014). Design and Use of Rubrics in Undergraduate Economics Courses. Perspectives on Economic Education Research, 9 (1), 36-55. 
Cano, E. (2015). La rúbrica como instrumento de evaluación de competencias en Educación Superior: ¿Uso o abusos? Profesorado. Revista de currículum y formación del profesorado, 19 (2), 265-280.

Castillo, S. y Cabrerizo, J. (2009). Evaluación educativa de aprendizajes y competencias. Madrid: Pearson.

Delgado, A.M. (Coord.), Borge, R., García, J., Oliver, R. y Salomón, L. (2003). Competencias y diseño de la evaluación continua y final en el Espacio Europeo de Educación Superior. Programa de Estudios y Análisis, Número de referencia: EA2005-0054. Ministerio de Educación y Ciencia, Dirección General de Universidades.

Domínguez, M., Martín, A.M., Paralera, C., Romero, E. y Tenorio, A.F. (2015). Evaluando con Webquest: Una experiencia en Matemáticas Financieras. Revista electrónica de Tecnología Educativa, 54.

doi: http://dx.doi.org/10.21556/edutec.2015.54

Espinosa, M.P. (2013). Evaluación de competencias mediante rúbrica. Importancia de las matemáticas en la evaluación de las competencias genéricas. Historia y Comunicación Social, 18. Número Extra 2 (Noviembre): La comunicación en la profesión y en la universidad de hoy, 243-255.

Fernández, A. (2010). La evaluación orientada al aprendizaje en un modelo de formación por competencias en la educación universitaria. Revista de Docencia Universitaria, 8 (1), 11-34.

Gámiz, V., Torres, N. y Gallego, M.J. (2015). Construcción colaborativa de una e-rúbrica para la autoevaluación formativa en estudios universitarios de pedagogía. Revista de Docencia Universitaria, 13 (1), 319-338.

García, M. P. (2014). La evaluación de competencias en Educación Superior mediante rúbricas: Un caso práctico. Revista Electrónica Interuniversitaria de Formación del Profesorado, 17 (1), 87-106.

Gimeno, J. (1988). El curriculum: Una reflexión sobre la práctica. Madrid: Morata.

González, J. y Wagenaar, R. (Eds.) (2006). Tuning Educational Estructures in Europe. Informe Final Proyecto Piloto-Fase II. La contribución de las universidades al proceso de Bolonia. Bilbao: Publicaciones de la Universidad de Deusto.

Goñi, J. M. (2008). El desarrollo de la competencia matemática. Barcelona: Graó.

Herbst, P. (2012). Las tareas matemáticas como instrumentos en la investigación de los fenómenos de gestión de la instrucción: Un ejemplo en geometría. Avances de Investigación en Educación Matemática, 1, 5-22.

Hull, M.M., Kuo, E., Gupta, A. \& Elby, A. (2013). Problem-solving rubrics revisited: Attending to the blending of informal conceptual and formal mathematical reasoning. Physical review Special Topics-Physics Education Research, 9, 010105. doi: 10.1103/PhysRevSTPER.9.010105

Ibarra, M.S., Rodríguez, G. y Gómez, M.A. (2012). La evaluación entre iguales: beneficios y estrategias para su práctica en la Universidad. Revista de Educación, 359, 206-231. doi:10.4438/1988-592X-RE-2011-359-092

Jonsson, A. (2014). Rubrics as a way of providing transparency in assessment. Assessment\& Evaluation in Higher Education, 39 (7), 840-852. doi:10.1080/02602938.2013.875117 
Jonsson, A. \& Svingby, G. (2007). The use of scoring rubrics: Reliability, validity and educational consequences. Educational Research Review, 2(2): 130-144. doi:10.1016/j.edurev.2007.05.002

Marin, J. A. y Santandreu, C. (2015) ¿Qué sabemos sobre el uso de rúbricas en la evaluación de asignaturas universitarias? Intangible Capital, 11(1): 118-145. doi:10.3926/ic.538

Martínez, M.E., Tellado, F. y Raposo, M. (2013). La rúbrica como instrumento para la autoevaluación. Revista de Docencia Universitaria, 11 (2), 373-390.

McGoldrick, K. \& Peterson, B. (2013). Using rubrics in economics. International Review of Economics Education, 12, 33-47. doi:10.1016/j.iree.2013.04.009

Mertler, C.A. (2001). Designing scoring rubrics for your classroom. Practical Assessment, Research \& Evaluation, 7 (25).

Ministerio de Educación, Cultura y Deporte (2013). Marcos y pruebas de evaluación de PISA 2012: Matemáticas, Lectura y Ciencias. Secretaría General Técnica. Subdirección General de Documentación y Publicaciones.

Panadero, E. \& Jonsson, A. (2013). The use of scoring rubrics for formative assessment purposes revisited: A review. Educational Research Review, 9, $129-144$. doi:10.1016/j.edurev.2013.01.002

Reddy, Y.M. y Andrade, H. (2010). A review of rubric use in higher education. Assessment \& Evaluation in Higher Education, 35(4): 435-448. doi:10.1080/02602930902862859

Roca, J., Valcárcel, M. y Verdú, M. (1990). Hacia un nuevo paradigma en la enseñanza de idiomas modernos. El enfoque por tareas. Revista Interuniversitaria de Formación del Profesorado, 8, 25-46.

Torres, J.J. y Pereda, V.H. (2010). La rúbrica como instrumento pedagógico para la tutorización y evaluación de los aprendizajes en el foro on-line en educación superior. Pixel-Bit, 36, 141-149.

Zabala, A. y Arnau, L. (2007). 11 ideas clave. Cómo aprender y enseñar competencias. Barcelona: Graó. 